Saudi Journal of Biomedical Research

Abbreviated Key Title: Saudi J Biomed Res ISSN 2518-3214 (Print) |ISSN 2518-3222 (Online)

\title{
Diagnostic Value of Red Cell Distribution Width (RDW), Mean Platelet Volume (MPV), and Platelets Count in Sudanese Neonates with Proven Sepsis
}

Albara Ahmed ${ }^{1 *}$, Ehab Mohammed Elmadenah Mohammed ${ }^{1,2}$, Babiker A. Mohammed ${ }^{3}$, Abdelhalim M Nasr ${ }^{4}$, Sahar ElderdiriGafar Osman ${ }^{5}$, Mosab Nouraldein Mohammed Hamad ${ }^{6}$

${ }^{1}$ Alfajr College for Science and Technology, Medical Laboratory Science, Department of Hematology, Khartoum, Sudan

${ }^{2}$ Faculty of Medical Laboratory Science, Department of Hematology, Dongola University, Dabba, Northern State, Sudan

${ }^{3}$ Karary University, Faculty of Medicine, Department of Pathology, Omdurman, Sudan

${ }^{4}$ University of Bahri, Faculty of Medicine, Department of Pediatrics, Khartoum North, Sudan

${ }^{5}$ Alfajr College for Science and Technology, Medical Laboratory science, Department of Histopathology and Cytology, Khartoum, Sudan

${ }^{6}$ Alfajr College for Science and Technology, Medical Laboratory Science Department, Phylum of Medical Parasitology, Khartoum, Sudan

DOI: $10.36348 /$ sjbr.2020.v05i07.001

| Received: 22.06.2020 | Accepted: 04.07.2020 | Published: 11.07.2020

*Corresponding author: Albara Ahmed

Abstract

Background: Sepsis is a life-threatening clinical condition that has cost humanity heavily since time immemorial. The aim of the study was to investigate the relationship between the red blood cell distribution width (RDW), mean platelet volume (MPV) platelet count, and neonatal sepsis in Sudan. Material and Method: A prospective cross sectional hospitalized based study was conducted in Omdurman maternity hospital, in the period from June 2013 to April 2015.Hundred Sudanese neonates were grouped equally into two; case group were neonates with proven sepsis by the culture $(\mathrm{N}=50)$, and control group were healthy neonates $(\mathrm{N}=50)$. Mean Platelet Volume (MPV), red blood cell distribution width (RDW), and platelet count were performed by using hematological analyzer (KX-21 Sysmex) and the results compared between two groups. Result: Of the fifty sepsis group 23 (46\%) were male, and 27(54\%) were female. Whereas in control group 24 (48\%) were male, and $26(52 \%)$ were female. The mean age in case group was 9 days, and in control group was 4.3 days. The mean of platelet count was significantly lower in sepsis group than control, $\mathrm{P}$ value $(<0.001)$. The mean of MPV and RDW CV were significantly higher in sepsis group than control, P. value $(<0.001$, and 0.026 respectively). In sepsis group, there were no significant change in MPV, RDW CV, and platelet count according to fate between died and recovered patient. Conclusions: The MPV and RDW were higher where as platelets count was lower in neonatal sepsis and these tests were inexpensive and commonly measured and may be with value in diagnosis and prognosis for neonatal sepsis. Further studies are required to determine the predicting value for MPV, RDW CV, and platelet count in neonatal sepsis.

Keywords: Red cell distribution width, mean platelet volume, platelet count, sepsis.

Copyright @ 2020: This is an open-access article distributed under the terms of the Creative Commons Attribution license which permits unrestricted use, distribution, and reproduction in any medium for non-commercial use (NonCommercial, or CC-BY-NC) provided the original author and source are credited.

\section{INTRODUCTION}

Sepsis is a global health problem that carries a high risk of death. A recent global assessment of the mortality rate of patients with sepsis treated in an intensive care unit found that over one third of these patients died without leaving hospital [1]. Sepsis remain the primary cause of death from infection despite advances in modern medicine, such as vaccine, antibiotics, and acute care [2]. In all countries where data on hospitalization for sepsis are available, the number of cases has increased steadily [3]. The number of hospital admission for sepsis following healthcareassociated as well as community-acquired infections increased up to three-fold over the last decade in comparison hospital admission for stroke and myocardial infarction remained stable over the same period [4]. In the developing world sepsis accounts for $60-80 \%$ of lost lives per year, affecting more than 6 million newborns and children annually and over 100,000 women contract sepsis in the course of pregnancy and childbirth [2]. Epidemiologic data in USA showed that the mortality of children with severe sepsis was $10.3 \%$, which can be up to $12.8 \%$ in children suffering from underlying diseases [5].

In Sudan locally, in 2010 deaths among children under five caused by $8 \%$ neonatal sepsis, 
neonatal mortality rate (the number of neonates dying before reaching 28 days of age, per 1000 live birth) was 29.9 in 2013, decreased from 2012 (30.5), and the neonatal mortality rate remained decreased annually since 2002 [6]. In 2013, sepsis represents the third cause of deaths in hospital. In Khartoum state, sepsis represents $12.6 \%$ from mortality causative agents, which higher mortality than heart diseases, malignant neoplasm, and respiratory system diseases in 2013. It is also one of the top 10 leading causes inpatient under five years, in 2013, for the under five years mortality, sepsis was the third causative agent $(10 \%)$, for a less than one year mortality, sepsis frequency was 658 deaths which more than seven fold mortality cases among 1-4 years for the same period [7].

Red cell distribution width (RDW) is a parameter reflecting the heterogeneity of the peripheral red blood volume and usually expressed with RDWcoefficient of variation (RDW-CV). In clinic, it can be understood whether the size of the red cell volume is uniform through detection of RDW. The more RDW is, the more uneven the red blood cell size is, and the higher the volume heterogeneity is. Recent studies found that RDW can take as a 'marker' of death in critical patients and can be used independently to predict the risk of death in critical patients [10]. New studies found that RDW increase can be used as an important and independent predictive factor of the incidence of deaths caused by various diseases. Meanwhile, RDW values can reflect the degree of overall inflammation and oxidative stress $[8,9]$. Luo et $a l$, at home found that RDW can predict the prognosis of critical patients [10]. Although the exact mechanism of the correlation between RDW and mortality of sepsis children is unclear, it may involve the changes of red cell balance caused by the body's inflammatory response and oxidative stress [11]. Increased RDW may comprehensively reflect the following pathophysiological mechanisms in occurrence and development of sepsis: a) inflammation may cause an increase of neuro-hormone and endocrine hormone in the body including noradrenaline, angiotensin 1 and other angiotensins level and renal ischemia. These neurotransmitters can stimulate red blood cell proliferation through promoting the generation of erythropoietin (EPO) to result in RDW increase [12]. b) Inflammatory factors may affect marrow hemopoietic function and iron metabolism in the body to cause RDW increase [13, 14]. c) RDW increase may indicate instable cytomembrane which may cause multiple organ dysfunctions to make the patients' condition deteriorate, thus leading to poor prognosis and increased mortality. Studies found that, the materials providing the nutrition to the body and cell, such as blood cholesterol, albumin, and others are also lack while RDW increases. Therefore, increased RDW may reflect the cell membrane instability due to the lack of cholesterol and other substances in the body $[15,16]$. Glomerular filtration rate (GFR) decreased progressively with increasing of RDW, and gastrointestinal dysfunction and liver function impairment may cause dysfunction of digestion and absorption to induce megaloblastic anemia or microcytic hypochromic anemia. Therefore, increase of RDW may reflect unevenness of red cell size due to liver function impairment- induced lack of hematopoietic elements (e.g. iron, folic acid, vitamin B12) in the body. A single or combined effect of the adverse factors above both can cause RDW increase, and RDW increase in sepsis newborns is likely caused by the combined action of several adverse factors [17]. RDW may become a new indicator for prognostic evaluation and risk stratification in sepsis newborns due to simple and easy detection, required lower expense, easily repeated measurement and convenient monitoring of outcome [18].

The mean platelet volume (MPV) describes the average size of platelets in a blood sample and is routinely measured by automated hematology analyzers using either electrical impedance or optical fluorescence method $[19,20]$. Increased in MPV during the first 72 hour of hospitalization is an independent risk factor for adverse clinical outcomes. Therefore, continuous monitoring of MPV may be useful to stratify mortality risk in patients with severe sepsis and/or septic shock [21]. The mean platelet volume (MPV) reflects the platelet size. Elevation of MPV is suggestive of increasing platelet production and activation. Larger platelets also contain more granules and prothrombotic materials [22, 23]. Rahul et al., suggest that high MPV on admission to the ICU with severe Sepsis suggests a higher chance of mortality during ICU stay [24].

In the present study, the RDW, MPV and platelet parameters, which are parts of a complete blood count, were assessed to evaluate the diagnostic efficiency of red blood cell distribution width (RDW), mean platelet volume (MPV), and platelets count for the prediction of sepsis.

\section{MATERIAL AND METHOD}

A prospective cross sectional hospitalized based study was conducted at Omdurman maternity hospital, in the period from June 2013 to April 2015. The cases were neonates with proven sepsis by the culture (case group). Patient were met the following criteria enrolled in case group; i) Septic neonate by positive blood culture, ii) age of 28 days or below, iii) not on vitamin $\mathrm{k}$ for last three days before sample collection, v) nor taking fresh frozen plasma or drug that interact with coagulation function for last two days prior to sample collection. A consecutive healthy neonate was taken as control for each case (control group). A neonate of age more than 28 days, negative blood culture, receive vitamin K, FFP, or drug interacts with test result, not admitted in Omdurman maternity hospital was excluded from case group. A neonate of 
age more than 28 days, not burn in Omdurman maternity hospital, clinically ill, or that has history of hereditary disorders was excluded from control group. After signing an informed consent, the Clinical and laboratory data (gender, mode of delivery, gestational age) was taken from each neonate (cases and controls) using questionnaire.

Venous blood samples were collected from neonates were collected in ethyle dehydrate tetra acetic acid (EDTA) for Cell blood count (CBC) studies ( $3 \mathrm{ml})$.RDW, MPV and Platelets count were done by use of automated haematology analyzer (KX21-Sysmix). The haematology analyzer KX21 counts platelets using the direct current detection method with coincidence correction automatic discriminators separate the cell population based on complex algorithm. The intensity of the electronic pulse from each analyzed cell proportional to the cell volume. Then platelet indices and red cell indices were calculated. Haematology analyzer switched on, reagents were checked, and control reagents used for calibration, samples were mixed by use of roller mixer (ERMA. Japan) for about 5 minutes then sample was introduced to the system probe then counted by haematology analyzer sysmexKX21, and results of RDW and MPV were calculated.

\section{Ethical Consent}

Ethical committee of Omdurman Maternity Hospital approved the study. Informed consent was obtained from parents of participated neonates.

\section{Statistical Analysis}

Data were entered in computer using SPSS version 20.0 (SPSS Inc, Chicago, IL, USA) for Windows. Continuous and categorized data were compared between the two groups (sepsis and health controls) using t-test, Mann-Whitney test, and Pearson chi square test $\left(\mathrm{X}^{2}\right)$, respectively, $\mathrm{P}<0.05$ was considered significant.

\section{RESULTS}

Fifty venous blood samples were investigated in each arm of the study. The demographic features were summarized in Table (1 and 2), 47 (47\%) were male, and 53(53\%) were female. In septic case group, 23 (46\%) were male, and 27(54\%) were female, whereas in healthy control group $24(48 \%)$ were male, and $26(52 \%)$ were female. The mean age in sepsis group per days was 9 (5.0), and in control group was 4 (2.9).The mean of platelet count was significantly lower in sepsis group than in control group $60.289 * 10^{9}(52.0)$ versus $212,030 * 10^{9}(62.6), \mathrm{P}$ value $(<0.001)$.The mean of MPV was significantly higher in sepsis group than in control10.6 (0.95) versus $8.8(0.85), \mathrm{P}$ value $(<0.001)$. The mean of RDW CV was significantly higher in sepsis group than control18.4 (2.1) versus (14.7 (1.8), P. value (0.026) (Table-3). According to the outcome of Study population, $10(20 \%)$ of sepsis group were died, and $40(49 \%)$ were recovered. There was no significant differences in MPV, RDW CV and platelet count between died and recovered patient (Table-4).

Table-1: Frequency (\%) of Gender in Study Population

\begin{tabular}{|c|c|c|c|}
\hline \multirow[t]{2}{*}{ Gender } & \multicolumn{2}{|c|}{ Study population Frequency (\%) } & \multirow[t]{2}{*}{ Total } \\
\hline & Case & Control & \\
\hline Male & $23(46)$ & $24(48)$ & $47(47 \%)$ \\
\hline Female & $27(54)$ & $26(52)$ & $53(53 \%)$ \\
\hline Total & $50(100.0)$ & $50(100.0)$ & $100(100)$ \\
\hline
\end{tabular}

Table-2: Mean (SD) of Age/ days in Study Group

\begin{tabular}{|l|l|l|l|l|}
\hline Age/days & $\mathbf{N}$ & Mean (SD) & Minimum & Maximum \\
\hline Case & 50 & $9(5.04)$ & 3.00 & 27.00 \\
\hline Control & 50 & $4(2.92)$ & 2.40 & 21.00 \\
\hline Total & 100 & $6(4.72)$ & 2.40 & 27.00 \\
\hline
\end{tabular}

Table-3: Comparison of platelet count mean platelet volume (MPV), platelet count, Red cell distribution width (RDW CV) between all groups

\begin{tabular}{|l|l|l|l|l|l|}
\hline \multicolumn{2}{|l|}{ Variables } & Mean (SD) & Minimum & Maximum & \multirow{2}{*}{ P. Value } \\
\hline \multirow{2}{*}{ Platelets count } & Case & $\mathbf{6 0 . 2 8 9}(\mathbf{5 2 . 0})$ & $\mathbf{8 . 0 0 0}$ & $\mathbf{2 5 5 . 0 0 0}$ & \multirow{2}{*}{$<0.001$} \\
\cline { 2 - 6 } & Control & $\mathbf{2 1 2 . 0 3 0}(\mathbf{6 2 . 6})$ & $\mathbf{1 2 5 . 0 0 0}$ & $\mathbf{4 2 1 . 0 0 0}$ & \\
\hline \multirow{2}{*}{ MPV } & Case & $\mathbf{1 0 . 6 0 4}(\mathbf{0 . 9 5})$ & $\mathbf{8 . 3 0}$ & $\mathbf{1 2 . 4 0}$ & \multirow{2}{*}{$<0.001$} \\
\cline { 2 - 5 } & Control & $\mathbf{8 . 8 9 4}(\mathbf{0 . 8 5})$ & $\mathbf{7 . 5 0}$ & $\mathbf{1 1 . 2 0}$ & \\
\hline \multirow{2}{*}{ RDW CV } & Case & $\mathbf{1 8 . 3 6 6 ( 2 . 1 )}$ & $\mathbf{1 4 . 7 0}$ & $\mathbf{2 2 . 8 0}$ & \multirow{2}{*}{$\mathbf{0 . 0 2 6}$} \\
\cline { 2 - 5 } & Control & $\mathbf{1 7 . 4 6 4 ( 1 . 8 )}$ & $\mathbf{1 5 . 0 0}$ & $\mathbf{2 3 . 0 0}$ & \\
\hline
\end{tabular}


Table-4: Distribution of Mean of Platelets, MPV, and RDW CV according to outcome of Study Population

\begin{tabular}{|l|l|l|l|}
\hline Variables & Fate & Mean & P. Value \\
\hline Platelets count $* \mathbf{1 0}^{\mathbf{9}} / \mathbf{l}$ & Died (10) & 61786.50 & 0.691 \\
\cline { 2 - 3 } & Recovered (40) & 54300.00 & \\
\hline \multirow{2}{*}{ MPV (fl) } & Died & 10.60 & \multirow{2}{*}{0.954} \\
\cline { 2 - 3 } & Recovered & 10.62 & \\
\hline \multirow{2}{*}{ RDW CV \% } & died & 18.22 & \multirow{2}{*}{0.343} \\
\cline { 2 - 3 } & Recovered & 18.94 & \\
\hline
\end{tabular}

\section{DISCUSSION}

The current study showed that, the mean of platelet count was significantly lower in septic neonates. This finding was in line with MS Ahmed et al., [25] who reported that thrombocytopenia is a common complication of neonatal sepsis, and WU et al., [26] who concluded that thrombocytopenia is prevalent in patients with severe sepsis. Also with Arif et al., [27] who found that thrombocytopenia is early predictor of septicemia, and De Stoppelaar et al., [28] who found that thrombocytopenia is a common finding in sepsis.

As has been shown before in different studies the reason could be due to that sepsis cause generalized inflammation and platelets are activated then been consumed in it. In fact, platelets count in septic neonates usually drop precipitously and recovery is often sluggish.

In present study, Red Cell Distribution Width (RDW) is significantly higher in septicneonate than in healthy. RDW is a parameter reflecting the heterogeneity of the peripheral red blood volume and usually expressed with RDW-coefficient of variation (RDW-CV). Recent studies found that RDW can take as a 'marker' of death in critical patients and can be used independently to predict the risk of death in critical patients ${ }^{(13)}$. Other studies found that increased RDW can be used as an important and independent predictive factor of the incidence of deaths caused by various diseases. Meanwhile, RDW values can reflect the degree of overall inflammation and oxidative stress [13], Lippi G et al., reported that RDW increased in sepsis [22]. Garofoli F et al., concluded that high RDW may become an indicator for prognostic evaluation and risk stratification in sepsis newborns due to simple and easy detection, required lower expense, easily repeated measurement and convenient monitoring of outcome [23].

In the existing study, MPV was significantly higher in sepsis neonate than healthy.Chan Ho Kim et $a l$., reported that increased in MPV during the first 72 hour of hospitalization is an independent risk factor for adverse clinical outcomes. Therefore, continuous monitoring of MPV may be useful to stratify mortality risk in patients with severe sepsis and/or septic shock [28]. Rahul et al., suggest that high MPV on admission to the ICU with severe sepsis, suggests a higher chance of mortality during ICU stay [24]. HJ, Park JT et al., concluded that: An increase in mean platelet volume from baseline is associated with mortality in patients with severe sepsis or septic shock [21].

\section{CONCLUSION}

The MPV and RDW were higher in sepsis neonate and platelet count was lower. These tests may become a new indicator for diagnostic, prognostic evaluation and risk stratification in sepsis neonate due to simple and easy detection, required lower expense, easily repeated measurement and convenient monitoring of outcome. Further studies are required to determine the predicting value for MPV, RDW CV, and platelet count in neonatal sepsis.

\section{RECOMMENDATIONS}

Further studies are required with other comparative diagnostic tools such as test CRP for septic neonates also engagement of other age groups is preferable.

\section{Conflict of interest: None}

\section{REFERENCES}

1. Vincent, J. L., Marshall, J. C., Namendys-Silva, S. A., François, B., Martin-Loeches, I., Lipman, J., ... \& Jimenez, E. (2014). Assessment of the worldwide burden of critical illness: the intensive care over nations (ICON) audit. The lancet Respiratory medicine, 2(5), 380-386.

2. Kissoon, N., Carcillo, J. A., Espinosa, V., Argent, A., Devictor, D., Madden, M., ... \& Global Sepsis Initiative Vanguard Center Contributors. (2011). World federation of pediatric intensive care and critical care societies: global sepsis initiative. Pediatric Critical Care Medicine, 12(5), 494-503.

3. Kaukonen, K. M., Bailey, M., Suzuki, S., Pilcher, D., \& Bellomo, R. (2014). Mortality related to severe sepsis and septic shock among critically ill patients in Australia and New Zealand, 20002012. Jama, 311(13), 1308-1316.

4. Seymour, C. W., Rea, T. D., Kahn, J. M., Walkey, A. J., Yealy, D. M., \& Angus, D. C. (2012). Severe sepsis in pre-hospital emergency care: analysis of incidence, care, and outcome. American journal of respiratory and critical care medicine, 186(12), 1264-1271.

5. Chen, J., Jin, L., \& Yang, T. (2014). Clinical study of RDW and prognosis in sepsis new borns. 
6. Annual health statistical report. (2012). National health information center. Sudanese ministry of health. 90-91.

7. Annual health statistical report. (2013). National health information center. Sudanese ministry of health. 84-91.

8. Hunziker, S., Stevens, J., \& Howell, M. D. (2012). Red cell distribution width and mortality in newly hospitalized patients. The American journal of medicine, 125(3), 283-291.

9. Bazick, H. S., Chang, D., Mahadevappa, K., Gibbons, F. K., \& Christopher, K. B. (2011). Red Cell Distribution Width and all cause mortality in critically ill patients. Critical care medicine, 39(8), 1913.

10. Marsh Jr, W. L., Bishop, J. W., \& Darcy, T. P. (1987). Evaluation of red cell volume distribution width (RDW). Hematologic pathology, 1(2), 117123.

11. Perlstein, T. S., Weuve, J., Pfeffer, M. A., \& Beckman, J. A. (2009). Red blood cell distribution width and mortality risk in a community-based prospective cohort. Archives of internal medicine, 169(6), 588-594.

12. Špinarová, L., Toman, J., Pospíšilová, J., Souĉek, M., Kára, T., \& Štejfa, M. (1998). Humoral response in patients with chronic heart failure. International journal of cardiology, 65(3), 227-232.

13. Chiari, M. M., Bagnoli, R., De Luca, P., Monti, M., Rampoldi, E., \& Cunietti, E. (1995). Influence of acute inflammation on iron and nutritional status indexes in older inpatients. Journal of the American Geriatrics Society, 43(7), 767-771.

14. Förhécz, Z., Gombos, T., Borgulya, G., Pozsonyi, Z., Prohászka, Z., \& Jánoskuti, L. (2009). Red cell distribution width in heart failure: prediction of clinical events and relationship with markers of ineffective erythropoiesis, inflammation, renal function, and nutritional state. American heart journal, 158(4), 659-666.

15. Chen, P. C., Sung, F. C., Chien, K. L., Hsu, H. C., Su, T. C., \& Lee, Y. T. (2010). Red blood cell distribution width and risk of cardiovascular events and mortality in a community cohort in Taiwan. American journal of epidemiology, 171(2), 214-220.

16. Pascual- Figal, D. A., Bonaque, J. C., Redondo, B., Caro, C., Manzano- Fernandez, S., SánchezMas, J., ... \& Valdes, M. (2009). Red blood cell distribution width predicts long- term outcome regardless of anaemia status in acute heart failure patients. European journal of heart failure, 11(9), 840-846.
17. Lippi, G., Targher, G., Montagnana, M., Salvagno, G. L., Zoppini, G., \& Guidi, G. C. (2008). Relationship between red blood cell distribution width and kidney function tests in a large cohort of unselected outpatients. Scandinavian journal of clinical and laboratory investigation, 68(8), 745748.

18. Garofoli, F., Ciardelli, L., Mazzucchelli, I., Borghesi, A., Angelini, M., Bollani, L., ... \& Merlini, G. (2014). The red cell distribution width (RDW): value and role in preterm, IUGR (intrauterine growth restricted), full-term infants. Hematology, 19(6), 365-369.

19. Jackson, S. R., \& Carter, J. M. (1993). Platelet volume: laboratory measurement and clinical application. Blood reviews, 7(2), 104-113.

20. Briggs, C. (2009). Quality counts: new parameters in blood cell counting. International journal of laboratory hematology, 31(3), 277-297.

21. Kim, C. H., Kim, S. J., Lee, M. J., Kwon, Y. E., Kim, Y. L., Park, K. S., ... \& Kang, S. W. (2015). An increase in mean platelet volume from baseline is associated with mortality in patients with severe sepsis or septic shock. PloS one, 10(3), e0119437.

22. Thompson, C. B., Jakubowski, J. A., Quinn, P. G., Deykin, D., \& Valeri, C. R. (1983). Platelet size as a determinant of platelet function. The Journal of laboratory and clinical medicine, 101(2), 205-213.

23. Rahul, P. N., \& Anita, S. P. (2018). Mean Platelet Volume and Its Outcome in Severe Sepsis-A Hospital Based Study. JMSCR, 6(3), 1210-1218.

24. Ahmad, M. S., \& Waheed, A. (2014). Platelet counts, MPV and PDW in culture proven and probable neonatal sepsis and association of platelet counts with mortality rate. $J$ Coll Physicians Surg Pak, 24(5), 340-4.

25. Wu, Q., Ren, J., Wu, X., Wang, G., Gu, G., Liu, S., .. \& Li, J. (2014). Recombinant human thrombopoietin improves platelet counts and reduces platelet transfusion possibility among patients with severe sepsis and thrombocytopenia: a prospective study. Journal of critical care, 29(3), 362-366.

26. Arif, S. H., Ahmad, I., Ali, S. M., \& Khan, H. M. (2012). Thrombocytopenia and bacterial sepsis in neonates. Indian Journal of Hematology and Blood Transfusion, 28(3), 147-151.

27. de Stoppelaar, S. F., van't Veer, C., Claushuis, T. A., Albersen, B. J., Roelofs, J. J., \& van der Poll, T. (2014). Thrombocytopenia impairs host defense in gram-negative pneumonia-derived sepsis in mice. Blood, 124(25), 3781-3790.

28. Rahul, P. N., \& Anita, S. P. (2018). Mean Platelet Volume and Its Outcome in Severe Sepsis-A Hospital Based Study. JMSCR, 6(3), 1210-1218. 